the "drift". Our predictions are that stroke deaths are likely to continue to decrease up to at least 2015. For 2015, our predictions of deaths from stroke varied from 34429 to 46538 (compared with 47213 deaths in 2005) under the different plausible model scenarios. We also noted that there were some potentially interesting trends in mortality amongst recent birth cohorts. In particular, mortality was higher in older age groups, but the difference between the older and younger age groups appears to have decreased over time for both sexes. Modelling of the subgroup aged 40-69 suggested a relative rate increase in mortality amongst those born since the mid-1940s compared with earlier cohorts; this trend appears to have been sustained in men.

Conclusions: Downward trends in stroke mortality are set to continue overall to at least 2015. However, these trends appear to be levelling off in middle-aged people, particularly men. This raises concerns that stroke mortality might increase as these cohorts grow older.

\section{CHOOSING METRICS IN PUBLIC HEALTH ASSESSMENTS: ATTRIBUTING CREDIT FOR THE RECENT LARGE CORONARY HEART DISEASE MORTALITY DECLINE IN THE US POPULATION}

${ }^{1} \mathrm{H}$ Gouda, ${ }^{2} \mathrm{JA}$ Critchley, ${ }^{1} \mathrm{~J}$ Powles, ${ }^{3} \mathrm{~S}$ Capewell. 'Department of Public Health and Primary Care, University of Cambridge, Cambridge, UK; ${ }^{2}$ The Institute of Health and Society, Newcastle University, Newcastle upon Tyne, UK; ${ }^{3} T h e$ Division of Public Health, University of Liverpool, Liverpool, UK

\section{doi:10.1136/jech.2009.096719d}

Background: Most explanations of falls in coronary heart disease (CHD) mortality limit themselves to event based metrics (such as fewer deaths). However, time based metrics (such as life-years gained (LYG)) promise to capture more of the social value attached to the deaths averted. We have assessed the sensitivity of conclusions about the relative contributions of treatments and risk factor changes to the choice of metric.

Methods: Using a validated CHD mortality model (IMPACT), we integrated data on the number of CHD patients, treatment uptake, treatment effectiveness, risk factor trends, and median survival among US adults aged 25-84 between 1980 and 2000, in order to estimate fewer deaths and LYGs. LYG were estimated using the US general population life expectancy for CHD onsets averted in 2000 and the median survival rates from Medicare for the additional survivors after CHD onset. (All the latter gains are currently attributed to treatments). We examined how uncertainty within the model may vary according to choice of metric.

Results: Between 1980 and 2000, CHD mortality-rates halved resulting in approximately 341745 fewer deaths in 2000; approximately $47 \%$ of the fall was attributed to treatments in patients (after clinical presentation) and $44 \%$ to population-wide risk factor reductions (independent of medication). However, this split was altered to $35 \% / 65 \%$ when LYG was used. Taking smoking as an example, those who did not experience a smoking attributable CHD death in 2000 because they did not smoke have been given the average US life expectancy (at the age of averted death) in 2000. The life-expectancy of the hypothetical non-smoker, however, would be expected to be higher. Applying these extended years to those deaths avoided by not smoking can add more than 50000 more life years attributable to the decline of smoking in the population. Furthermore, while the model attributes all gains from increased survival post CHD onset to improved treatments, an increasing proportion of non-smokers among CHD patients could result in additional LYG attributable to smoking reduction.

Discussion and Conclusions: Using life-years gained rather than deaths avoided strengthens the case for primary prevention by risk factor reduction, because it captures more fully the social gains that result. The additional assumptions required are all relatively minor. It seems that past versions of the model may understate the relative importance of risk factor changes when better assessed using the metric of life-years gained.

\section{EXPLAINING THE MASSIVE DECLINE IN CORONARY HEART DISEASE MORTALITY IN ICELAND BETWEEN 1981 AND 2006}

${ }^{1} \mathrm{M}$ O'Flaherty, ${ }^{2} \mathrm{~T}$ Aspelund, ${ }^{2} \mathrm{~V}$ Gudnason, ${ }^{2} \mathrm{BT}$ Magnusdottir, ${ }^{3} \mathrm{~K}$ Andersen, ${ }^{4} \mathrm{G}$ Sigurdsson, ${ }^{2} \mathrm{~B}$ Thorsson, ${ }^{5} \mathrm{~J}$ Critchley, ${ }^{6} \mathrm{~K}$ Bennett, ${ }^{1} \mathrm{~S}$ Capewell. ${ }^{1}$ Department of Public Health, University of Liverpool, Whelan Building, Quadrangle, Liverpool, UK; ${ }^{2}$ Icelandic Heart Association, Kopavogur, Iceland; ${ }^{3}$ University of Iceland, Reykjavik, Iceland; ${ }^{4}$ Landspitali University Hospital, Reykavik, Iceland; 5 Institute of Health and Society, University of Newcastle, Newcastle, UK; ${ }^{6}$ Department of Pharmacology and Therapeutics, Trinity Centre for Health Sciences, St James's Hospital, Dublin 8, Ireland

\section{doi:10.1136/jech.2009.096719e}

Background: Coronary heart disease mortality rates have been decreasing in Iceland since the 1980s. We used the validated IMPACT model to examine how much of the $80 \%$ decrease in Iceland between 1981 and 2006 could be attributed to medical and surgical treatments and how much to changes in cardiovascular risk factors.

Methods: The previously validated IMPACT mortality model was used to combine and analyse data on uptake and effectiveness of cardiological treatments and risk factor trends in the entire Iceland population. The main data sources were official statistics, national quality registers, published trials and meta-analyses, clinical audits and a series of national population surveys. Sensitivity analyses were then conducted.

Results: Between 1981 and 2006, coronary heart disease mortality rates in Iceland decreased by $80 \%$ in men and women aged 25 to 74 years. This fall resulted in 295 fewer deaths in 2006. Approximately one quarter of this decrease (27\%) was attributable to treatments in individuals (including some $11 \%$ to secondary prevention, $5 \%$ to heart failure treatments, $5 \%$ to initial treatments of acute coronary syndrome, $2 \%$ to hypertension treatments and less than $1 \%$ to lipid reduction). Almost three quarters of the mortality decrease $(72 \%)$ was attributable to population risk factor reductions (principally cholesterol, 35\%; smoking, 22\%; systolic blood pressure, $22 \%$ and physical activity, $5 \%$ ). Adverse trends were seen for diabetes $(-5 \%)$, and obesity $(-4 \%)$.

Conclusions: Almost three quarters of the large coronary heart disease mortality decrease in Iceland between 1981 and 2006 was attributable to reductions in major cardiovascular risk factors in population (mainly decreases in total serum cholesterol, smoking and blood pressure levels). Very little was attributable to primary prevention medications. These findings emphasise the value of a comprehensive strategy that promotes tobacco control and a healthier diet. It also highlights the potential importance of effective, evidence based medical treatments.

\section{20-YEAR TRENDS IN MAJOR CORONARY RISK FACTORS IN OLDER BRITISH MEN: ASSESSING THE IMPACT OF MEDICATION USE}

${ }^{1} \mathrm{SL}$ Hardoon, ${ }^{2} \mathrm{PH}$ Whincup, 'SG Wannamethee, 'O Papacosta, 'MC Thomas, ${ }^{1} \mathrm{LT}$ Lennon, ${ }^{1} \mathrm{AG}$ Thomson, ${ }^{3} \mathrm{~S}$ Capewell, ${ }^{1} \mathrm{RW}$ Morris. ${ }^{1}$ Department of Primary Care 8 Population Health, Division of Population Health, UCL Medical School, University College London, Royal Free Campus, Rowland Hill Street, London, UK; ${ }^{2}$ Department of Community Health Sciences, St George's, University of London, Cranmer Terrace, London, UK; ${ }^{3}$ School of Population Community \& Behavioural Sciences, Division of Public Health, University of Liverpool, Whelan Building (3rd Floor), Brownlow Hill, Quadrangle, Liverpool, UK

\section{doi:10.1136/jech.2009.096719f}

Background: Favourable trends in blood pressure (BP) and blood lipids have contributed to the falling incidence of coronary heart disease (CHD) in recent decades. The role of medication in the BP and blood lipid trends is unknown. 